\title{
ABENTEURER AM HOFE KAISER LEOPOLD I.
}

(Alchemie, Technik und Merkantilismus)

\section{VON HEINRICH RITTER VON SRBIK}

Es gibt keinen Zweig der Wissenschaft, für den der Weg zur Erkenntnis nicht durch das Land des Irrtums geführt hätte. An der Wiege der Astronomie stand die Astrologie, an der Wiege der Chemie die Alchemie. Jahrhundertelang ist sie die Herrin der Chemie geblieben, dann hat des großen Paracelsus Lebensarbeit die Chemie zur Dienerin der Medizin gemacht, und neben dieser Begrenzung ihrer Ziele blieb ihre Hauptaufgabe das Suchen nach dem Steine der Weisen, bis endlich gegen den Ausgang des siebzehnten Jahrhunderts durch Robert Boyle die Erkenntnis sich Bahn brach, daß die Chemie um ihrer selbst, um der Er. forschung der Natur willen, nicht zur Edelmetallerzeugung zu pflegen sei. $\left.{ }^{1}\right)$ Robert Boyles Lehre von den Elementen hat die erste große Bresche in den Schutzwall der Alchemie gebrochen, Generationen aber sind noch vergangen, bis seit Lavoisier die Chemie zur wirklich selbständigen Herrscherin geworden ist und ihrer Nebenbuhlerin den Rest gegeben hat.

An den Furstenhöfen drängten sich die Adepten und andere Jünger der werdenden Naturwissenschaft, die merkantilistische Wirtschaftspolitik offnete ihnen Tür und Tor. Ihre Bahn konnte zur Höhe der Fürstengunst und zum schmachvollsten Lose führen: gelangen ihre Versuche oder schienen sie wenigstens zu gelingen, dann wartete ihrer höfische Gnade, Adel und Reichtum; wurden sie als Betrüger entlarvt, dann sahen sie an mehr als einem ihrer Vorgänger, daß ihrer Kerker und Elend, vielleicht sogar der goldflitterumsponnene Galgen harrte.

Es ist eine stattliche Reihe von österreichischen Herrschern, die den geheimen Künsten ihre Gunst zugewandt haben, die sich teils selbst im Laboratorium versucht haben, teils wenigstens den Jünern der ersehnten Wissenschaft Schutz und Förderung angedeihen ließen; Alchemie und Astrologie, damals noch so

) Herm. Kopp, Die Alchemie in alterer und neuerer Zeit, I T. (Heidelberg I886), S. 52 f. 
innig verbunden mit ihren spateren Verderbern Chemie und Astronomie, haben lange Zeit am österreichischen Hofe eine Heimstätte gefunden, und ,seit undenklichen Zeiten“", heißt es noch I783, "war Wien so wie einst Florenz der Tummelplatz aller geldsüchtigen Alchemisten, die von den vier Winden der Erde hier zusammentrafen und in öffentlichen und Privat. gebäuden weitläufige Laboratorien errichteten."

Von Maximilian II. und Ferdinand von Tirol, dessen Unterstützung ein Thurneysser genoß ${ }^{2}$ ), von Rudolf II., dem ,deutschen Hermes Trismegistos", der in Prag einen Mittelpunkt naturwissenschaftlichen Strebens schuf, von Ferdinand III. und Leopold I. bis zu dem Lothringer Franz I, und dem letzten bedeutenden Adepten Sehfeld ${ }^{3}$ ) standen alle unter dem Banne einer Idee. Wenn Tycho Brahe und Kepler, die nebst Galilei und Baco von Verulam die eigentlichen Träger der neuen, großen Ideen waren, an Rudolf einen warmen Förderer fanden, so sind doch anderseits auch die Namen derer, die unter dem Deckmantel der Wissenschaft den Kaiser in die Irre leiteten, - eines Kelley, Güstenhöver, Sendivogius, Müller von Müllenfels und anderer - außerordentlich zahlreich. Am Hofe Leopold I. fehlten die großen Baumeister des Gebäudes der Naturwissenschaften fast ganz, die Handlanger fanden sich in reichster Menge ein.

Ein Zug geistiger Verwandtschaft, die Leopold I. mit Rudolf II. verbindet, liegt in dieser Vorliebe fur das chemische Experiment. Auf der Wasserkunstbastei in Wien ${ }^{4}$ ) wurde das neue Laboratorium des Kaisers erbaut, das im Jahre I 674 vollendet und I 675

1) Vgl Alex Bauer, Chemie und Alchemie in Österreich bis zum beginnenden I9. Jahrhundert (Wien 1883), und derselbe, Die Adelsdokumente österreichischer Alchemisten (Wien I 89.3), auch für das unmittelbar folgende.

${ }^{7}$ Kopp, a a. O. S. 109 ff ; vgl auch J. Hirn, Erzherzog Ferdinand II. von Tirol, 1. Bd., S. 36 ıf.; Herm. Schelenz, Geschichte der Pharmazie (Berlın 1904), S 24I.

3) Kopp a. a. O., 2 T, S $223 \mathrm{ff}$.

4) Diese Bastei wurde I 545 errichtet, I860 demoliert und lag zwischen der Braunbaster und der Kärntnerbastion, nahe dem Kärntnertor, das I67I erbaut wurde. Vgl K. Weiß, Geschichte der Stadt Wien, 2 Aufl, 2. Bd I W'ien I883), S. 320 f. und W. Kisch, Die alten Straßen und Plätze Wiens (Wien 1883), S. 318 In den Munzgraben hinter der Wasserkunstbastei wurde nach 1677 die Münzstátte verlegt (vgl K Schalk, Numismat Zeitschr. N F. I. Bd., S 16f.). Abbildung bei Kisch S. 336. 
mit den notigen Chemikalien und Geräten versehen war. ${ }^{1}$ ) Auch in diesem kaiserlichen Laboratorium hat gar häufig trügerische Gewinnsucht ihr Handwerk getrieben: sei es, daß die Kunstler einfach unedles goldscheinendes Metall unterschoben oder Goldoxyd, Goldamalgam und andere Goldpräparate der Masse beimengten oder unechte Munzen vergoldeten, wie jener Johann Wenzel Seiler, der 1675 seine Kunst, Kupfer und Zinn in Gold zu wandeln, zeigte und fur seine vermeintlichen Verdienste mit

1) Über die Einrichtung des neuen Laboratoriums Leopolds I. unterrichten Akten, die im Hofkammerarchive in Wien, Hoffinanz, Fasc. No. 13835 , liegen; die Ausgaben vom 18 Mai 1674 bis einschließlich I 5 Januar 1675 betrugen $3000 \mathrm{fl}$., 26 Mark fein Gold und 60 Mark fein Silber (Passierungsbefehl an das Hofzahlamt 6. April I675). Aus den zahlreichen beiliegenden Rechnungen geht hervor, $\mathrm{da} \beta$ der in der historischen Literatur nicht unbekannte Alchemist Johann Wenzel Seiler die Ausstattung des Laboratoriums leitete, daselbst seine Experimente trieb und wohl als Famulus des Kaisers bezeichnet werden kann. Die Empfangsbestatigungen erstrecken sich auf alles Erforderliche: Seiler quittiert uiber Geld, fein Gold und fein Silber, Quecksilber, Blei und Salz, verschiedene Professionisten quittieren uber die Bezahlung von gelieferten Ziegeln, Platten, Treibscheiben, Öfen, Schmelzgeschirren, Tiegeln, Retorten, „Zimentbuichsen" und der verschiedenartigsten Gläser (Fünferkolben, Achterkolben usw.), Helmstücke, Rezipienten, Phiolen, Trichter; es finden sich ferner Rechnungen des Tischlers für Gestelle $u$. a., des Faßbinders, des Schneiders (darunter schwarze Leinwand für Fürtucher und Kappen, schwarze Zwilchlaborantenröcke, blaue Kappen mit ,blechernen Augengläsern"), des Zimmermalers, des Schlossers fur Destillieròfen, Glocken, Hämmer zum Silberschlagen, des Klempners, Deckenmachers, des Steinmetzen für geschliffene Märbelsteine und marmorne Reibsteine; der Materialist Johan Bapt. Aquilino verrechnet Salpeter, Vitriol, Leinol, Arsenik (Auripigment), Sulphur, Zinnober, Rauschgelb, Federweiß, Kobalt, „Margalita “ (= Markasit?), rot und weiß Galmei, Silbertalg, Blutstein, Ungar. Vitriol, Grünspan, Terra sigillata rot und weiß, Bolus Armena, Borax, Salmiak, Alaun, Weinstein, Potasche, Baumwolle u. a., ferner Vitriol und Salpeter zum Brennen von Scheidewasser. Schließlich fehlen auch die Rechnungen von Maurern über das Setzen von Brenn-, Destillier- und Schmelzofen und diverse kleinere tägliche Bedürfnisse nicht. Die Bediensteten des Laboratoriums verdingte Seiler, ihr Lohn betrug nach der Liste Seilers vom 30 . Juli bis 17 . Dezember 1674 wöchentlich $30 \mathrm{fl}$. $30 \mathrm{kr}$., ein bekannterer Name findet sich, soweit ich urteilen kann, nicht darunter. Unter den Dienern und Tagelohnern zu erwähnen sind wohl nur ein Johann Georg Gebel aus Sontra in Niederhessen, der für Schmelzen, Abtreiben und Zementieren der Metalle wöchentlich 3 fi. bezog, ferner Gottlieb Riedl von Spitz in Niederösterreich mit $4 \mathrm{fl}$. fur Scheiden und Zementieren und zur Sorge fur die "philosophischen" (i. e. alchemistischen) 
dem Adelsdiplom belohnt wurde ${ }^{1}$ ); sei es, daß durch gewisse Legierungen unedler Metalle dem Produkte die Farbe der Edelmetalle verliehen wurde, wie namentlich die Legierung von Kupfer mit Arsen oder Zink und Amalgamierung von Kupfer sehr beliebt gewesen $z u$ sein scheint.2) Die geheimnisvollsten Krafte schrieb man dem rätselhaften Quecksilber $z u$, in dem schon der mythische Araber Geber, der Vater der Alchemie, neben dem Sulphur den Mercurius, den zweiten Grundstoff der Metalle und die Ursache ihres Glanzes, ihrer Dehn- und Schmelzbarkeit und Fähigkeit zur Amalgamation vorzüglich enthalten $\mathrm{sah}^{3}$ ); Quecksilber ,fixieren“, in feuerbeständiges hartes Metall verwandeln zu konnen, haben seit dem dreizehnten Jahrhundert bis an die Schwelle des neunzehnten gar viele behauptet.4) Oft glaubte man auch eine Verwandlung vor sich $\mathrm{zu}$ haben, wo nur eine Scheidung vorlag, wenn es gelang, aus edelmetallhältigen Substanzen, beispielsweise Goldamalgam enthaltendem Quecksilber, durch Destillieren, Sublimieren und andere

Öfen, endlich Friedrich Adam Rzynski, ein Apotheker aus Öls in Schlesien, mit $3 \mathrm{fl}$. Wochenlohn für „Brennung des subtilen Spiritus zu Medizin und andern vorfallenden Destillierungen". Zahlreiche Abbildungen von Destillationsgefäßen, Brennöfen u. a. siehe bei Herm. Peters, Aus pharmazeutischer Vorzeit in Wort und Bild (Berlin 1886), S. I1 3 ff. Über die bekannte Vorliebe Leopolds fur Alchemie vgl. Rink, Leopolds des Großen wunderwürdiges Leben und Thaten (Cölln I7I3), S. II $8 \mathrm{f}$; dazu auch A. Dimitz, Zur Geschichte der Alchemie in Krain, Mitteilungen des Histor. Vereins für Krain, Dezember 186r. In der Wiener Hofbibliothek liegt übrigens ein gänzlich unverwertetes, umfangreiches und interessantes "Gutachten Joh. Joach. Bechers über Herrn Daniels Marsaly Prozeß zur Tinktur", das Becher auf Verlangen Leopolds verfaßte und dem Kaiser am II. Mai 1674 in Laxenburg uiberreichte (Msk. Nr. II 472).

1) Vgl. C. Chr. Schmieder, Geschichte der Alchemie (Halle 1832), S. 445 f.; A Bauer, Chemie und Alchemie, S. $38 \mathrm{ff}$. und Kopp, a. a. O. S. I58f. Wie weit der Ruf Seilers drang, geht aus emem Brief Robert Boyles an Joseph Glanville vom I8. September 1677 hervor, worin er erwähnt, der kaiserliche Minister Graf Waldstein habe ihm versichert, that the famous frier Wencel has several times actually made transmutations of baser metals into gold in the presence of the emperor and divers noblemen and good chemists. (The works of the hon. Robert Boyle, ed. by Th. Birch, 6. Bd., London 1772, S. 58.) 9) Kopp, S. 159.

3) Über Geber s. H. Kopp, Beiträge zur Geschichte der Chemie, 3. Strick (Braunschweig I874), S. I 3 ff.

4) Kopp, Gesch. der Alchemie, I T., S. $248 \mathrm{ff}$. 
chemische und mechanische Operationen den Edelmetallgehalt abzusondern.

Trotz aller Enttäuschungen des Kaisers fanden die Versucher bei Leopold I. immer wieder ein williges Ohr; wie er jenen Johann Konrad von Richthausen, Freiherrn von Chaos, der vor Ferdinand III. Quecksilber in Gold verwandelt hatte, zum Oberstkammergrafen in den oberungarischen Bergstädten ernannte ${ }^{1}$ ) und von dem erwàhnten Seiler sich 20000 fl. entlocken ließ; so hat vor ihm ein Johannes de Monte Snyders Blei veredelt, und der Italiener Francesco Guiseppe Borri, eine der abenteuerlichsten Gestalten, der behauptet, den Kaiser vor Vergiftung gerettet zu haben und aus Quecksilber Gold und Silber bereitete, will Leopold bis zu Tränen gerührt haben, als ihn der Nuntius nach Rom ins Gefängnis abforderte ${ }^{2}$ ); eine bunte Kette, deren Glieder größtenteils noch unbekannt, teils auch kaum wert sind, der Vergessenheit entrissen $z u$ werden, und unter denen sich doch der eine oder andere findet, dessen Schicksale als typisch für die Zeit und wegen seiner Verbindung mit hervorragenden Persönlichkeiten Beachtung verdienen.

Die Bodenproduktion Österreichs gab den alchemistischen Bestrebungen hier besonderen Antrieb; bot Idria mit seiner in Europa fast konkurrenzlos dastehenden Quecksilbergewinnung den Adepten ihren bevorzugten Stoff in reichster Menge, so gewährten anderseits namentlich auch die oberungarischen Bergwerke Kremnitz, Schemnitz und Neusohl mit ihrem Reichtume an Gold, silberhaltigen Erzen und Kupfer ein Versuchsfeld, in das die Regierung denn auch fast jeden, der sich der geheimen Künste rühmte, zur Probe sandte. Die in Schemnitz angestellten

1) Vgl. Schmieder, a. a. O. S. 397 ff., Ad. Wrany, Geschichte der Chemie und der auf chemischer Grundlage beruhenden Betriebe in Böhmen Prag r9o2), S. 32 f. und Schelenz, a. a. O. S. 250; ferner mein Buch, Der (staatliche Exporthandel Österreichs von Leopold I. bis Maria Theresia (Wien und Leipzig 1907), S 36 A. I. Über die Experimente Chaos' siehe auch des bekannten Merkantilisten Wilhelm von Schröder Unterricht vom Goldmachen (Ausgabe v. J, I728 in Roth-Scholtzens Theatrum Chemicum), S. 232 f.; daselbst auch eine ausfuhrliche Erzählung über Wagnerecks Experimente.

2) Vgl. A. Bauer, a. a. O. S $49 \mathrm{f}$ und "Adelsdokumente" S. $28 \mathrm{f}$; Schelenz, a. a O. S. $252 \mathrm{f}$ 
Versuche, die Oxyde der leichten Metalle zu reduzieren, wie sie aus dem ausgehenden achtzehnten Jahrhundert bekannt sind $\mathbf{1}$ ), finden weit über ein Jahrhundert vorher schon zahlreiche Vorláufer; neben ihnen stehen ungezählte Projekte, die Aufbereitung der Metalle zu verbessern und verbilligen; namentlich die Zementkupfererzeugung in Schmölnitz hat schon seit Paracelsus als Beweis der Möglichkeit von Metallverwandlungen gegolten ${ }^{2}$ ), und durch Jahrhunderte hat diese Art, mittels der Korrosion des Eisens durch kupferhaltiges Wasser Kupfer zu gewinnen, dem kleinen ungarischen Bergstädtchen Weltruhm verschafft ${ }^{3}$ ) und zu Versuchen mit anderen Metallen Anregung gegeben. In den ungarischen Bergwerken mußten auch die meisten Vertreter des naturwissenschaftlichen Treibens ihrer Zeit, die dem Hofe ihre Kunst anboten, ihr Wissen beweisen. Zwei der bezeichnendsten solcher Episoden mögenzur Schilderung dieses Treibens dienen.

Als einer der genialsten Diplomaten seiner Zeit, Franz von Lisola, im Jahre 1672 seinen ununterbrochenen Kampf gegen Ludwig XIV. im Haag fuhrte und nach fast unüberwindlichen Schwierigkeiten das Bundnis Kaiser Leopold I. mit der von Frankreich angegriffenen Republik der Vereinigten Niederlande zustande brachte, trat ein gewisser Jean Baptiste Liefrinck, erster Kommis der Amsterdamer Westindischen Kompanie ${ }^{4}$ ),

1) A. Bauer, Chemie und Alchemie, S. $6 \mathrm{r}$.

$\Rightarrow$ H. Kopp, Geschichte der Chemie, 4. Bd. (Braunschw. I847), S. I63f. und A. Bauer, a. a. O. S. I $2 \mathrm{ff}$.

3) Bezeichnend hierfur ist auch ein Brief John Lockes an Robert Boyle vom 12./22. Dezember I 665 , Cleve, worin er ihm uber die merkwürdige Verwandlung von Eisen in Kupfer berichtet, die bei Neusohl im Kupferbergwerke vor sich gehe, und von der ihm ein Jesuit erzảhlte, der in Ungarn gewesen und zum Gefolge des kaiserlichen Gesandten Baron Goess gehörte (Boyles Works VI, 536).

4) Lisola an den Hof kanzler Baron Hocher, I6. Februar I673, Haus-, Hof- und Staatsarchiv, Hollandica. Das hervorragende genealogische Werk von J E. Elias, De vroedschap van Amsterdam (Haarlem I 903 bis I905, 2 Bde.) I. Bd, S. 463 fuhrt als dritten Gatten der Elisabeth Wijmer (getraut I. April I670) an Jan Baptista Liefrinck, van Leiden, commis over de coopmanschappen van de Westindische Compagnie, Regent spin- en werkhuis I 673 , wonende op 't Nieuwe Eiland, wedr van Elisabeth Kamps. Aus der Ehe Liefrinck-Wijmer gingen keine Kinder hervor, der junge Jean Baptiste Liefrınck durfte wohl ein Sohn aus der Ehe des Genannten mit Elisabeth Kamps gewesen sein. 
mit einem vielversprechenden Vorschlage an ihn heran: er habe eine einfache Maschine erfunden, mittels deren man aus dem Sande der Berge, Ebenen oder Flusse das ganze darin enthaltene Gold ohne Verlust eines einzigen Granes und mit viel geringerem Aufwande an Arbeitskraften, Geld und Zeit, als der gewöhnliche Prozeß erfordere, ziehen kónne; er sei geneigt, dem Kaiser seine Erfindung zu uberlassen. ${ }^{1}$ ) Es war naturlıch klar, daß der Mann auf Gewinn ausging, doch deuteten schon seine Stellung und der Umstand, daß er keinen Geldvorschuß forderte, darauf hin, daß man es mit keinem der gewóhnlichen Großsprecher zu tun habe, und Lisola, der ja uberhaupt beweglichen Geistes und rasch entflammt war, ließ sich vóllig gefangen nehmen. Die einflußreichsten Persönlichkeiten des Kaiserhofes, der Hofkanzler Hocher, der Hofkammerprásident Georg Ludwıg Graf Sinzendorf, forderten Lisola auf, Sorge zu tragen, daß das Geheimns des Antragstellers keinem anderen Fursten in die Hände falle 2 ); die Herzöge von Braunschweig wollten Liefrinck tatsächlich für sich gewinnen, Lisola suchte ihn vergeblich zu bewegen, nach Wien zu reisen; schließlich erklarte er sich bereit, dem Diplomaten personlich seine Kunst zu offenbaren. Am 14. Februar 1673 schlossen beide im Haag mit Vorbehalt der kaiserlichen Rati. fikation cinen Vertrag, daß Liefrinck Lisola seine Erfindung demonstrieren, ihm ein vollkommenes Modell der Maschine in die Hand geben und ihn in deren Gebrauch unter der Bedingung unterweisen werde, daß dem Erfinder nach Einführung der Maschine in die Praxis der kaiserlichen Erblande ein Achtel des allenthalben gegenüber dem gewöhnlichen Aufwande erziclten Ersparnisses zufalle, und daß fur ihn bereits nach Gebrauchnahme des Instrumentes aut Verrechnung monatlich 2000 Rtl. bei der Bank von Amsterdam eingezahlt und $\mathrm{hmm}$ jahrlich uberdies ein Fuder Wein gegeben werde; Lisola verpflichtete sich, die Erfullung dieser Forderungen und die Ratifikation des Kaisers

1) Kopie der Proposition bei Schreiben Lisolas an Hocher 9. Februar 1673 , Hof kammerarchiv, Hoffinanz, Fasc. Nr. I3829.

9) Auszug aus dem Schreiben Hochers an Lisola 16. Oktober und Sinzendorfs an denselben 27. Oktober 1672 bei 18. Juli 1674, Hof kammerarchiv, Ungar. Münz- u. Bergwesen, Fasc. Nr. 15289. 
zu erwirken. Eine Verringerung der Vergutungen sollte zu keiner Zeit, auch nicht unter dem Vorwande einer neuen Erfindung, statthaben; erfolgte die Ratifikation nicht, so war Lisola ehrenwortlich zum Stillschweigen verpflichtet, wie anderseits Liefrinck während der Dauer des Vertrages keiner anderen Partei sein Ge. heimnis bekanntgeben durfte. ${ }^{1}$ ) Nach Abschluß dieses Kontraktes trennte Liefrinck vor Lisolas Augen in einem Augenblick Goldstaub von dem Sande, mit dem er ihn gemischt hatte, der Gesandte erklärte das Mittel fur untruglich; wenn größere Maschinen gebaut wurden, konnten an einem Tage ungeheuere Mengen Goldes durch einen einzigen Mann vom Staube gereinigt werden ${ }^{2}$ ); der Kaiser sagte, hiedurch bewogen, die geforderte Entschädigung zu und versprach seine Ratifikation. ${ }^{3}$ )

Es mögen in Wien dann doch Bedenken aufgestiegen sein, ob man sich nur auf Lisolas Zeugnis hin, ohne selbst die Erfindung kennen gelernt und erprobt $z u$ haben, zu so weitgehenden Verpflichtungen mit unbeschränkter Dauer herbeilassen solle. So sandte denn Liefrinck seinen Sohn Jean im Juli 1673 nach Wien, Empfehlungen Lisolas an Hocher, Sinzendorf und den kaiserlichen Beichtvater Muller geleiteten ihn. ${ }^{4}$ ) Lisola scheint ihm, selbst wenn das Instrument sich als nicht brauchbar erweisen sollte, eine sichere Stelle bei Hofe versprochen zu haben. Voll froher Hoffnungen auf Ehre und reichen Gewinn trat der junge Mann seine Reise an, Monate später erst traf er nach den größten Fährlichkeiten, seiner Habe beraubt und fast mittellos, an seinem Bestimmungsorte ein. ${ }^{5}$ )

1) Kopie des Kontraktes bei Hofkammerdekret vom 18. Juli I674, Hofkammerarchiv, Ungar. Münz- und Bergwesen, Fasc. Nr. I5 289.

*) Lisola an Hocher I6. Februar und II März 1673, Staatsarchiv, Hollandica.

3) Weisung an Lisola 4. Mảrz 1673, Hof kammerarchiv, Hoffinanz, Fasc. Nr. 13829.

4 Lisola an Sinzendorf und an Hocher 18. Juli 1673, Brissel, und 28. September 1673, Köln, ebenda, Ungar. Münz- und Bergwesen, Fasc. Nr. 15289 .

5) Das Folgende nach den Akten in Ungar. Munz- und Bergwesen, Fasc. Nr. 15289 . - Als Jean Liefrinck von Amsterdam nach Köln reiste, wurde er von den Franzosen gefangen genommen und seines Gepácks und Reisegeldes beraubt, der Prinz Condé suchte ihn, als er Lisolas Empfehlungsschreiben las, fur Ludwig XIV. zu gewinnen, Ludwig selbst 
Ein trauriges Los wurde ihm zuteil; die ganze Tragik der täglichen Lebensnot eines ehrenhaften Mannes, der, dem väterlichen Hause entrissen, um einer verfehlten Sache willen die fremde Gnade anflehen und das harte Brot der Mildtätigkeit eines enttäuschten Hofes genießen muß, spricht aus seinen Hilferufen an den Hofkammerpräsidenten. Hocher und Pater Müller schickten ihn zu Sinzendorf, Sinzendorf an den Hofkammersekretär Mayer, jeder erklärte, er könne nichts für ihn tun, Unterstützungen seitens des allmächtigen Präsidenten, die mehr einem Almosen glichen, genügten nicht einmal zum täglichen Unterhalt $\left.{ }^{1}\right)$, das kostspielige Leben in Wien mehrte Liefrincks Schulden von Woche zu Woche, vergebens bat er um eine monatliche feste Summe oder wenigstens eine bescheidene Stelle, un petit coing à la coure de $\mathrm{V}^{\mathrm{re}} \mathrm{Exc}^{\mathrm{ce}}$, zu der ihn seine Kenntnis der englischen, französischen, deutschen und holländischen Sprache und seine mehrjährige Erfahrung in der Buchführung befähigten. Im Februar 1674 war Liefrinck bereits so niedergedruckt, daß er auf den Anspruch des achten Gewinnteiles und der monatlich zugestandenen 200 Dukaten verzichten und nur einen Gehalt von 50 Dukaten sechs Monate lang beziehen wollte ${ }^{2}$ ); seine Bitten blieben ebenso

soll sich für sein Geheimnis interessiert haben; nach fast sechswöchiger Gefangenschaft wurde er freigelassen und kam Ende September nach KöIn, wo Lisola, auf dem bekannten Friedenskongresse tätig, der durch die Gefangennahme des Fürstenbergers ein jähes Ende nahm, ihm wieder die größten Versprechungen machte. Von seınem Vater neu ausgestattet, fuhr Liefrinck weiter und wurde zwischen Mainz und Frankfurt von den Kaiserlichen iberfallen und abermals ausgeplündert; am 29. Oktober 1673 nahm er bei den Kaufleuten Pierre und David Neufville in Frankfurt Geld auf und kam endlich nach einem Verluste von insgesamt etwa 500 Dukaten in Wien an (Liefrincks Orig.Schreiben an Sinzendorf 2. und 19. April 1674).

1) Liefrinck an Sinzendorf exp. 29 Dezember 1673, 2. April, 19. April, 29. Juni 1674. (Je n'ay pas un sous à achepter un pair de soulliers ... Je n'ay pas le moyen de me pouvoir faure faire un habit ni rien .. Je suis un garçon d'honneur et non pas accoustumé à vivre en une poverté comme on me laisse ici ... véritablement, Monselgneur, je n'avois jamais cru d'estre traitté ici en telle manière, cela n'encourage pas les gens à faire les services à sa $\mathrm{M}^{\mathrm{t} e} \mathrm{Imple}$... Je ne cherche point une vie pompeuse, mais honneste et modeste. . Ce m'est plus pour l'honneur que pour l'argent etc )

2) Representations aux illustres seigneurs de la chambre aulique de sa Mte Imple r. Februar 1674. 
unerhört, wie sein Gesuch, die Hofkammer möge ihm wenigstens die Freiheit, mit anderen Interessenten abzuschließen, gewähren.1) Die ganze brutale Rucksichtslosigkeit eines Sinzendorf, der selbst das Ärar um Millionen betrog, aber einen durchaus ehrlichen, wenn auch vom Erfolge nicht beglückten Mann - er hatte ihm mit Kavaliersehrenwort seine Förderung versprochen - im Stiche ließ, fiel auf Liefrinck.

Ich möchte nicht mit Sicherheit behaupten, daß die Erfindung wirklich unbrauchbar war. Es handelt sich ja wohl nur um eine rasche und gründliche Scheidung des Goldes aus goldhaltigem Sande auf mechanischem Wege. Allein dieses Instrument bewährte sich zum mindesten für die österreichischen Verhältnisse entschieden nicht; die ersten Proben zeigten das Modell als nicht sehr geeignet, auf neue Modelle aus Amsterdam wartete Liefrinck anscheinend vergebens. ${ }^{2}$ ) Einen wesentlichen Anteil an dem Mißlingen dürfte aber auch die geringe Goldhaltigkeit des zu den Proben verwendeten Sandes gehabt haben.

Die Hofkammer hatte von verschiedenen Orten an der Donau, wo vormals Goldwäscherei betrieben worden war, Berichte und die Einsendung einiger Zentner goldhaltigen Wellsandes gefordert. $^{3}$ ) In der Nähe von Ungarisch - Altenburg wurde noch von wenigen Leuten, die das Gold gegen Entschädigung von io Fl. für das Lot der Herrschaft übergeben mußten, Donausand gereutert und mittels Quecksilbers das wenige darunter befindliche Edelmetall herausgezogen; die Tagesausbeute zweier Männer betrug kaum eine Quantität von der Größe einer Linse oder Erbse. Bei Purkersdorf war das Goldwaschen seit einigen Jahren wegen Geringhältigkeit des Donausandes überhaupt aufgegeben worden, in Wörth bei Eblsberg trug einem Goldwäscher die Arbeit des Tages IO-30 Kreuzer ein, andere Orte kamen bei hohem Wasser-

1) Liefrinck an Mayer I3. Februar I674.

$\Rightarrow$ Liefrinck an Sinzendorf 29. Dezember 1673, 19. April 1674 .

s) Hofkammer an die Mautbeamten zu Linz und Ybbs, die Proviantverwalter zu Raab und Komorn und die Beamten des Wiener Waldamtes 15 . Februar und 6. Juni I674, Hofkammerarchiv, Niederösterr. Münzu. Bergwesen, Fasc. Nr. 17327. 
stande überhaupt kaum in Betracht. ${ }^{1}$ ) Unter solchen Umständen hätte wohl auch ein besser geeignetes Instrument wenig Erfolg gefördert. So blieb denn noch die große Probe in den ungarischen Bergstädten, die Liefrinck retten und ihm wenigstens zu einer „billigen Rekompens", - so viel nur hatte er von den hochfliegenden Erwartungen bewahrt - helfen konnte. ${ }^{2}$ )

Nach zwei Jahren, wahrend derer der Schutzling Lisolas, durch den Tod seines Protektors jeder Stutze beraubt, in Schemnitz und Kremnitz wiederholt arbeitete, hatte sich auch hier die gänzliche $Z$ wecklosigkeit weiterer Bemuhungen herausgestellt. Liefrinck selbst sah ein, daß sein Werk gescheitert sei. ${ }^{3}$ )

Sein Vater suchte seine Kunste am Goldsande der Kuste von Guinea zu verwerten, gründete eine Kompanie und soll bei der Überfahrt vom Kapitän im Streite erschlagen worden sein, ,,und seinen Sohn", schreibt Johann Joachim Becher, ,habe ich in Österreich gesehen, welcher sich mit einem Waschwerk schleppet. Dieses ist der traurige Ausgang von so einem köstlichen Konzept. " 4 )

Neben diesen Typus des ehrlich von seiner Kunst überzeugten und durch ihre Unzulänglichkeit selbst am schwersten betroffenen Mannes möchte ich den des Abenteurers im schlimmen Sinne stellen, bei dem Geldgier das einzig treibende Moment ist, und dem die Voraussetzungen zu wissenschaftlichen Fortschritten ebenso

1) Berichte des Administrators von Ungar. Altenburg Johann Haaß von Hochburg $s$ d., des Waldmeisters Zacharias Adam von Baursperg und des Waldschaffers Christoph Schreyer s. d, der Ybbser Mautbeamten 10. März und 27. Juni I674, der Linzer Mautbeamten 13. März 1674, a. a. O. Ungar. Muinz- und Bergw., Fasc. Nr. 15289 . - 1682 stand dann das Gesuch eines Michael Poll in Beratung, der um Verleihung des Privilegs, in der Donau, Mur, Save und andern Flussen allein Gold waschen zu dürfen, gebeten hatte (Niederösterr. Buchhaltereibericht exp. 30. Juni 1682, a. a. O. Hoffinanz, Fasc. Nr. 13 858.). Über die Wiedereinfuhrung der Goldwäscherei unter Karl VI. vgl. Notizenblatt d. histor.-statist. Sekt d. Mähr.-schles Gesellsch. 1877 , S. $4 \mathrm{ff}$.

2) Hofkammerdekret an das Hofzahlamt und Hofk.-Schreiben an die Oberbeamten in Schemnitz und Kremnitz 18.Juli 1674, a. a. O. Fasc. 15289.

s) Liefrinck an Sinzendorf exp. 28. Juli 1676 und Hotk. an den Administrator in den ungar. Bergstädten vom selben Tage, Liefrinck $150 \mathrm{fl}$ zu geben, ebd. Fasc. Nr. 1529 I.

4) Närische Weißheit und weise Narrheit (Ausgabe von 1707), S. I57 f. Nr. 36 "Liefrincks Invention, um Gold aus dem Sand in Guinea zu bohren." 
wie die Errungenschaften selbst, die er erzielt zu haben behauptet, und gewiß auch der eigene Glaube an seine Fähigkeit fehlen. Unter den vielen, die dieser Spezies angehören, greife ich eine der interessantesten Persönlichkeiten heraus, die über zwei Jahre lang den Kaiserlichen Hof getáuscht, gewaltige Summen Geldes verbraucht und den Schutz Leopolds I. selbst genossen hat. Es ist der kaiserliche Oberst ${ }^{1}$ ) Wolfgang Ferdinand Freiher $r$ von Schellenberg. Sein Name ist nicht völlig unbekannt, in den Beschreibungen des Becherschen Manufakturhauses auf dem Tabor findet sich eine Schellenbergsche Schmelzhütte erwähnt, von der jedoch außer einem Berichte über den „Universalofen " bisher nichts Näheres bekannt war. ")

Ein rauher, unumgänglicher Mann war dieser Schellenberg; seit etwa funfundvierzig Jahren in kaiserlichen Kriegsdiensten, dem Trunke ergeben, zum Wortstreite und Degenziehen stets bereit, selbst voll Unkenntnis und Unbildung und doch gleich gewillt, andere, die seine Fehler rügten, des Betruges und der Parteilichkeit zu zeihen. Als altgedienter Oberst bot er dem Kaiser im Jahre $1674^{3}$ ), wie bereits Jahre vorher, gegen Zusicherung eines Funftels des Nutzens oder eine hohe Belohnung seine Kunst an, aus dem Schlich die ,fluchtigen sulphurischen, arsenikalischen

1) Gerade Militärs, denen die notwendigsten technischen und chemischen Kenntnisse fehlten, traten nicht selten mit Projekten hervor; bezeichnend ist da namentlich der Fall eines Soldaten vom Salmschen Regiment, der in Spanien gelernt haben wollte, wie man bei Bergwerken mit geringen Kosten und wenig Arbeitern die Grubenwässer heben könne; die Probe in einem Teiche in Schemnitz fiel nicht schlecht aus; als aber die Maschine in den Gruben versucht werden sollte, verschwand der Mann, nachdem er iiber 2000 fi. Kosten verursacht hatte, und die Versuche ergaben die völlige Unbrauchbarkeit seiner Methode (Hofkammer an den Oberstkammergrafen v. Viechter I6. Mai I684, Bericht desselben I Juli I 684, Hofkammerarchiv, Ungar. Munz- u. Bergwesen, Fasc. Nr. I 5 297.)

2) Vgl. J. Hatschek, Das Manufakturhaus auf dem Tabor in Wien, Schmoller's Staats- u. sozialwiss. Forschungen VI $/ \mathrm{r}$, S. $37 \mathrm{f}$; Abbildung und Beschreibung des Schellenbergschen Ofens in Schröders Unterricht vom Goldmachen a. a. O. S. $282 \mathrm{ff}$.

3) Das Folgende nach Akten im Hof kammerarchive, Ungar. Münz- u. Bergwesen, Fasc. Nr. I5 289, 15290 . - Schellenberg hatte auch schon Ferdinand III. und Erzherzog Leopold Wilhelm Anträge gestellt, die ", arglistig gefunden wurden". (Hofk.-Referat exp. 20. Mai I676, ebd, Hoffinanz, Fasc. Nr. I3 I39.) 
und koboldischen Erze zu fixieren und zu schmelzen". Auf Betreiben Sinzendorfs wurde das Anerbieten angenommen und Schellenberg alsbald nach Ungarn gesandt. Proben, die er noch im selben und im folgenden Jahre in Schemnitz ablegte, führten nur zu Streitigkeiten mit den Bergbeamten, so daß wegen der schweren Beschuldigungen, die Schellenberg erhob, sogar das Berggericht eingreifen mußte. Trotz der bedeutenden Auslagen und des schon offensichtlichen Mißerfolges ließ Leopold den Obersten nicht fallen, es wurde im Manufakturhause in Wien, das im März I676 im ganzen vollendet war, eine eigene vollkommen ausgerüstete Schmelzhütte fur Schellenberg erbaut ${ }^{1}$ ), und zu Beginn des Jahres 1676 wurde ihm Gelegenheit geboten, in Wien seine Kunst durch die Tat zu beweisen; sollte es ihm gegenüber dem alten Verfahren gelingen, das Doppelte an Silber mit seiner Fixier- und Schmelzmethode zu erzielen, so hatte er Anspruch auf eine Summe von 100 ooo Rtl., die in jährlichen Raten von Io 000 Rtl. aus dem erzielten Mehrgewinne gezahlt werden sollte; fur ein noch besseres Resultat wurde eine noch höhere Belohnung in Aussicht gestellt, im Falle des Mißlingens sollte Schellenberg die Kosten tragen. ${ }^{2}$ ) Erz und Schlich, Schmelzer und Maurer aus Kremnitz wurden nach Wien gebracht, Schellenberg versprach dem Kaiser jährlich eine Million Mehrertrag. Vergeblich wies die Hofkammer darauf hin $^{3}$ ), daß in Kremnitz während eines ganzen Jahres nicht mehr als 500 Mark Silber aus dem Schlich und Schlamm mit 4-5 Prozent Schmelzabgang gewonnen würden, daß also, wenn dieser Abgang durch die Fixierkunst erhalten werden solle, Jahre bis zum Mehrgewinn auch nur einer Million vergehen müßten, selbst wenn das Doppelte an Silber gewonnen würde; vergeblich machte sie auf die höheren Kosten des Schellenbergschen Verfahrens und auf den schlechten Ausgang der in den Bergstädten abgelegten Proben wie auf die augenscheinliche Gewinnsucht des Obersten aufmerksam, der, von seinen Gläubigern bedrängt, schon nach der ersten Probe die to o0o Rtl. erhalten

1) Die Bau- und Einrichtungskosten der Schmelzhtitte betrugen $243 \mathrm{I}$ fl. $5 \mathrm{Kr}$.

2) Versicherungsdekret 2 I. Jänner I676, a. a. O. Fasc. Nr. I529I.

3) Hof kammerreferat ebd. 
wollte. Der Kaiser befahl die Abhaltung neuer Proben in Wien ${ }^{1}$ ), und eine Kommission, deren Mitglied auch der berühmte Merkantilist Johann Joachim Becher war, wurde zur Aufsicht bestellt. $^{2}$ )

Mit einem der Kommissare, dem Hofkammerrate Thomasis, kam Schellenberg alsbald in solchen Konflikt, daß beide zu den Waffen griffen ${ }^{3}$ ); die neue Kommission, bestehend aus den Hofkammerräten v. Seeau, v. Vestenburg, Becher und dem Münzwardein Sigmund Hammerschmidt4), kam gleich nach den ersten Proben in der Wiener Schmelzhütte zu einem vernichtenden Urteile. Ganz abgesehen von dem unerträglichen Benehmen des Obersten zeigte sich in den Proben, die vom IO. Februar bis 18. März I 676 dauerten, und während deren er gegen den Zimenter und den Wardein die unbegründetsten Beschuldigungen erhob, ,daß er wohl etwas von Zeitigung der Erze mag gehört, aber die Kunst (des Fixierens und Schmelzens) nicht verstanden noch begriffen habe"; er brannte beim Schmelzen die Blasbälge an, rostete den Lech zu stark und bewies „daß weder das Schellenbergische Mischen der Erze noch deren Fixierung noch sein Schmelzen noch sein Rösten noch sein Abtreiben, mit einem Worte seine ganze Scienz in dieser Sache nichts nutz seien", daß seine Fixierkunst „vielmehr eine Destruierkunst sei", und daß anstatt einer Willion Nutzen eine Million Schaden in den Bergwerken entstehen kónnte. Er hatte versprochen, 34 Lot Silber zu erzeugen, tatsächlich brachte er nur 2 Lot zuwege und richtete dabei unter einem Aufwande von 5-6000 Fl. noch beträchtlichen Materialschaden an. ${ }^{5}$ ) Nun konnte man vielleicht, wie es Schellenberg tat, der Kommission Voreingenommenheit vorwerfen und auch auf das Urteil der Kremnitzer Schmelzer nicht zu viel Gewicht legen, die erklärten, Schellenberg habe fast sechs Wochen zu

1) Eigenhändige Bemerkung Leopolds. Ich wil in alleweg diese Prob gemachter wissen, in übrigen wie angerathen, Leopold.

2) Hotkammerdekret 28. Dezember I675, a. a. O. Fasc. Nr. 15290

3. Schreiben Schellenbergs an Sinzendorf ca. Februar und Bechers 3. Februar 1676 , a. a. O. Hoffinanz, Fasc. Nr. I 3839.

4) Über Hammerschmidt vgl. K.Schalk, Beiträge zur Geschichte des österr. Munzwesens unter Leopold I., Numismat. Zeitschrift N. F. I., S. I9

5) Referat der Kommission vom März 1676 , a. a. 0 . 
einer Arbeit gebraucht, die in Kremnitz in dreizehn Tagen vollzogen werde, sein Werk sei von Anfang an unsauber gewesen und verrate vollkommene Unerfahrenheit. ${ }^{1}$ ) Unbestreitbar trotz aller Ausfluchte des „Künstlers" blieb, daß eine nach dem alten Kremnitzer Verfahren in Wien angestellte Probe ein um zwei Drittel größeres Silberresultat aus der gleichen Menge Erz und Schlich mit bedeutend geringeren Kosten ergab. ${ }^{2}$ )

Wieder wäre die Zeit gekommen gewesen, dem Schellenbergschen Unfuge ein Ende zu machen; die Kommission hatte schon im Februar geraten, die großen Proben des alten und neuen Verfahrens nicht in Wien, sondern in den mit den geeigneten Hochöfen, dem passenden Material und geubten Arbeitern versehenen Bergstädten vornehmen zu lassen. Nachdem doch in Wien die Versuche durchgeführt worden waren und ein für Schellenberg so klägliches Ergebnis gezeitigt hatten, beantragte die Hofkammer wieder, ihn nunmehr ein für allemal abzuweisen, auch jetzt aber ließ Leopold den Mann nicht fallen. ${ }^{3}$ ) Als neuerliche kostspielige Proben abermals kein verläßliches befriedigendes Resultat ergaben, Schellenberg sich auf den Ausweg des passiven Widerstands verlegte und die Hofkammer endlich anriet, ihn in Arrest zu setzen und zur Vornahme der geforderten Versuche zu zwingen, erklärte Leopold, er verlange wohl der Sache auf den Grund zu gehen, könne aber aus gewissen Grunden diesen Vorschlag noch nicht billigen; auf ausdrücklichen Befehl des Kaisers wurde der Oberst im Herbste 1676 abermals in die Bergstädte geschickt, und dort dürfte sich wohl endlich der Zusammenbruch seines haltlosen Gebäudes vollzogen haben ${ }^{4}$ ), das nur durch des Kaisers

1) Protokoll über die Aussage der Schmelzer.

2) Gutachten der Kommission 13. April, Hofkammerreferat exp. 20 Mai I676.

s) Das zitierte Hofk.-Referat; eigenhändige Bemerkung Leopolds: Weillen durch der Commissarien Relation der Schellenberg zimblich gravirdt und fast angeklagt wirdt, hingegen niemandt inauditus $z \mathfrak{u}$ con. demnieren, als köndte dem v. Schellenberg der Comissarien Relation in substantia communicierdt, sein Verandtwordtung vernommen und sodan uber ein und anderes von der Hofcammer Ihr Gutachten geben werden. Indessen solle man ihm in $3 \mathrm{mal}$ mitt $300 \mathrm{fl}$. helffen Leopold. Entsprechendes Hofk.-Dekret an die Kommissaire 20. Mai 1676.

4) Akten unter d. 20. Juli und 2. September I 676, Ungar. Muinz- u. Bergw., Fasc. Nr. 1529 I. 
Leichtgläubigkeit und Hartnäckigkeit so lange gestützt worden war.

Eine Rolle eigentümlicher Art hat Becher in der Schellenbergschen Affäre gespielt. In den offiziellen Berichten der Kommission fällte er mit den anderen Delegierten das härteste Urteil über den Erfinder, in seinen an Sinzendorf persönlich gerichteten Einzelgutachten äußerte er sich zunächst keineswegs so ungünstig, da er wußte, daß Schellenberg vom Hofkammerpräsidenten selbst ermuntert worden war, suchte aber den Rivalen durch noch größere Versprechungen zu uberbieten und auszustechen. Noch im Mai 1676, als er selbst gegenuber der Kammer schon über Schellenberg abgeurteilt hatte, erklärte er Sinzendorf gegenüber die Vorschläge des Obersten als prinzipiell durchaus möglich und beachtenswert, die Art der Operation wolle er weder verwerfen noch billigen; zugleich erbot er sich aber selbst, aus dem ungarischen Schlich nicht allein doppelt, sondern zwölfmal so viel Silber als auf gewöhnlichem Wege zu gewinnen; seine Kunst und Erfahrenheit könne, auch wenn actualiter im Schlich nur $1 / 2$ Lot Silber enthalten sei, doch den potentialiter darin steckenden größeren Gehalt lösen; auch die Kunst, aus jedem C. Blei $3^{\circ}$ Lot Silber ${ }^{1}$ ) und aus jedem C. Quecksilber IoO Lot Silber herauszubringen, also „die wahrhafte Kunst der Verbesserung und Zeitigung der Metalle" sei ihm zu eigen. ${ }^{2}$ ) Wenige Wochen später bezeichnete er wieder in einem offiziellen Gutachten Schellenbergs Fixierungstheorie als den Naturgesetzen widersprechend und scheute sich nicht, den Mann als Betrüger hinzustellen, zugleich seine eigene Kunst nochmals anzubieten. ${ }^{3}$ )

Ich wollte damit nur an einem bezeichnenden Einzelfalle dartun, wie sehr man sich hüten sollte, auf Grund von Bechers ver-

1) Die Abbildung einer Múnze, deren Silber Becher 1675 aus Blei erzeugt haben will, bei A. Bauer, Die Adelsdokumente österr. Alchemisten. Über einen Kontrakt Bechers mit dem Bischofe von Lavant, wöchentlich aus einer Mark Feinsilber einen Dukaten Gold zu gewinnen, ohne daß der Gewichtsverlust Schaden bringe, vgl G. Karschulin, Streiflichter zur österr. Gewerbegeschichte, Wochenschrift des niederösterr. Gewerbevereins 1898 , S. 17.

2) Becher an Sinzendorf, Hoffinanz, Fasc, Nr, I 3839 .

s) Gutachten 5. Juni 1676, ebd. 
öffentlichten Schriften allein ein Bild seines Wesens zu zeichnen ${ }^{1}$ ), und wie sehr selbst diesem Manne, der doch als erster Begrunder der Phlogistontheorie in der Geschichte der Chemie einen ehrenvollen Platz einnimmt ${ }^{2}$ ), dessen Lehre bis zur Entdeckung der Gase die Anerkennung der Besten genoß, und in dessen physikalischen und chemischen Erfindungen und Projekten so viel ernste Überzeugung und redliche Tat steckt, ein Zug des Schwindelhaften anhaftet, jener Zug, der so vielen seiner Zunft ihr eigenartiges Gepräge gibt.

Noch lange nach Schellenbergs mißgluckten Versuchen und Bechers Scheiden aus Österreich hat die Hofkammer beide als Musterbeispiele von Leuten hingestellt, die viel versprachen, aber nichts leisten konnten, den Kaiser straflich hinters Licht geführt und um viele tausend Gulden geschädigt haben. ${ }^{3}$ ) Trotzdem wurde man durch den Schaden nicht klug; der Wiener Hof blieb eine bevorzugte Zufluchtsstätte der Adepten, die neue Metallschmelzverfahren erfunden haben ${ }^{4}$ ), die rauhen und flüchtigen Erze fixieren ${ }^{5}$ ), aus Quecksilber und Schwefel Gold und Silber gewinnen ${ }^{6}$ ), das arcanum metallurgicum ${ }^{7}$ ), das aurum

1) Wie es R.v.Erdberg-Krczenciewski, Johann Joachim Becher(Staatswissenschaftliche Studien herausgeg. v. L. Elster, 6. Band, 2. Heft, 1896), getan hat.

2) Vgl. H Kopp, Geschichte der Chemie 3. Bd (Braunschweig 1845), S. $108 \mathrm{ff}$. und Beiträge zur Geschichte der Chemie 3. Stück, S. $202 \mathrm{ff}$.

9) Hotbuch haltereibericht 16 . September, Hotkammerreferat exp. r6. Oktober I68I, Ungar. Munz- u. Bergw., Fasc. Nr. ${ }_{5} 295$.

4) In den Jahren 1686-1688 erregten die Verheißungen eines gewissen Laurentius Zignaga große Hoffnungen, der ein neues Verfahren für das Kupferschmelzen einführen wollte; seine Methode scheint sich bei den nur mit Eisen und Blei vermengten schwedischen Erzen bewährt, bei dem Neusohler Kupfererz, das Schwefel, Vitriol, Kies und Antimon enthielt, als unanwendbar erwiesen zu haben (ebd. Fasc. Nr. 15298 und 15 299); kaum hatte sich dies herausgestellt, so meldete sich ein Franciscus Maria Levanto mit einem ähnlichen Verfahren und wurde wieder zugelassen (Ungarn, Fasc. Nr. I 4 659).

5) So der Sohn eines Augsburger Münzmeisters Christian Holeysen 1688-1692, der den Goldgehalt des Schemnitzer Silbers niederschlagen wollte, und dessen Verfahren sich gleichfalls als unpraktisch erwies (Ungar. Munz- u. Bergwesen, Fasc. Nr. 15299 und I 5300 )

") Auf Somers und Zaparelli habe ich in meinem "Staatl.Exporthandel etc." S. I 3 kurz hingewiesen; sehr eingehende, nicht uninteressante Nachrichten uiber Somers bringt Schröders Unterricht vom Goldmachen, a. a. O.S. $240 \mathrm{ff}$.

7) 1697 erhielt ein Anonymus, der das Geheimnis kennen wollte, unbrauchbare Bergwerke wieder zu großer Ausbeute zu bringen, fur den 
potabile ${ }^{1}$ ), die Tinktur zur Multiplizierung der Edelmetalle besitzen wollten. ${ }^{2}$ ) Alle Abstufungen von der brauchbaren Erfindung bis zur bewußten betrügerischen Vorspiegelung, von der tatsächlichen Errungenschaft bis zum absolut Unmöglichen finden sich in den ungezählten Vorschlägen, aus denen ich hier nur eine kleine Blütenlese gegeben habe ${ }^{3}$ ).

Neben diesen Physikern undChemilern, Alchemisten undGeheimkunstlern, steht die fast unübersehbare Reihe jener Männer, die, den volkswirtschaftlichen Zug der merkantilistisch denkenden Zeit und zugleich das finanzpolitische Interesse des Staates erfassend, ihre Heilmittel dem Kaiser anboten. Die Einkünfte des Staates waren ja durchaus unzulänglich, Österreich lebte, teilweise auch infolge der elenden Finanzverwaltung, von der Hand in den Mund; Jahr für Jahr zerbrachen sich die Hofkammerrate den Kopf, wie durch

Fall guten Erfolges die Oberstbergwerksdirektorstelle in Iglau, Schenkung von einigen kaiserlichen Dörfern im Werte von 70000-80000Fl.u. a. zugesichert (Hofdekret an den Franziskaner Alipio Reuter, Hoffinanz, Fasc. Nr. 13905).

1) Es ist der wahre Stein der Weisen. - Eingabe des Mathias Cornelius Höll, er habe das aurum potabile gefunden, das seinen Effekt nicht nur an den Menschen erzeigen, sondern auch alle unvollkommenen Metalle zur Vollkommenheit bringen soll, so zwar, daß diese Metalle dem rechten und natürlichen Golde und Silber keinen Vorzug gönnen; das "mercurialische Wasser" sei der einzige Schlussel, mit dem der Körper der Metalle aufgelöst werden könne, längstens in sechs Wochen wolle er das aurum potabile herstellen. Hofkammer an den Oberstkammergrafen Thavonat 5. Januar I7 14, Höll zu dieser Probe $1000 \mathrm{fl}$. zu geben (Ungar. Münz- u. Bergwesen, Fasc. Nr. 15308).

2) Noch 1735 bot ein Dr. Jeremias Fester an, die von ihm zur Vermehrung des Goldes und Silbers erfundene Tinktur dem Kaiser zu überlassen; Proben wurden bewilligt (Hofk, an den Münzmeister Mittermayer v. Waffenberg I r. Juni I 735, Niederösterr. Münz- u. Bergw., Fasc. Nr. I7332).

") Besonders reichhaltig war das Programm eines Grafen Ferdinand Gottlieb Voikra, der 1682 um Privilegien und Belohnung für folgende Erfindungen bat: 1. Wasserquellen in den Bergen zu erwecken, wo keine sind; 2. das Wasser aus der Tiefe der Bergwerke mit geringen Kosten $\mathrm{zu}$ erheben; 3. mit emem neuen Instrumente sine tabula sinuum vel tangentium die Perpendikurlarhöhe oder Tiefe der Berge und die lineam horizontalem zu messen; 4. mit geringen Kosten ohne Windschacht frisches Wasser in die Tiefe der Bergwerke zu bringen Die nied.-österr. Buchhalterei und der Oberstkammergraf betonten die Nützlichkeit dieser Erfindungen, falls sie sich bewähren sollten, nur aus Geldmangel wurden keine Remunerationen, wohl aber Proben bewilligt, die Volkra vielleicht gar nicht versucht hat (Hotkammerreferat und Hofdekret an Volkra 22. Juni I682, Ungar. Münz- und Bergw,, Fasc. Nr. I 5296). 
„extraordinari Mittel“ die dringendsten Bedürfnisse angesichts des ständigen Defizits, des fortwährenden Überwiegens des Ausgabenetats über denEinnahmenetat in den unzuverlässigenVoranschlägen, gedeckt werden könnten. Die Turkensteuer und allerlei Aufschläge, $Z$ wangsanleihen und Belastung der letzten Fonds haben in diesen Verhältnissen ihren Ursprung genommen. Namentlich die Einführung der Akzise von allen Konsumtibilien, die Kopfsteuer von Menschen und Vieh, die Beschreibung der Vermögen und eine entsprechende Anlage, der Verkauf der Steuer in Niederösterreich, das sind die gewöhnlichsten Mittel, die von der Hofkammer immer wieder vorgeschlagen wurden, gutenteils aber infolge der Gegnerschaft der Stände nicht zur Durchführung gelangten. Mochten die Projekte der sich Herandrängenden zur Erhöhung der Regalien, zur Vermehrung der Intraden der Länder noch so phantastisch sein, mochten sie noch so sehr das Zeichen der Undurchführbarkeit an der Stirne tragen, selten wurde einer a limine abgewiesen: von Leuten, die "gewisse ungehobene Schätze" verraten wollten ${ }^{1}$ ), von dem römischen Anonymus, der einen Ritterorden mit finanzieller Grundlage, den Titel- und Ämterschacher, unterwertige Münze, Stempelpapier, Lotto und Leihbank vorschlug'), bis zu jenen Einsichtsvolleren, die in einer vernünftigen Volkswirtschaftspolitik die Rettung sahen, auch

1) Hofdekret an Paravicino von Paravicin 28 Dezember 1674 , Hoffinanz, Fasc. $\mathrm{Nr} \mathrm{I}_{3} 8_{34}$ Ähnliche Vorschlage wiederholt, so das Anerbieten eines gewissen Johannes Marcius Roverela, Hofdekret 31 . August 1675, ebd. Fasc. Nr. I 3836 Im Jahre 1677 erbot sich der Venezianer Bartholomeo Biasii, ein Mittel, „die Paradeyß-Regel genannt", bekannt zu geben, das dem Kaiser in wenigen Stunden viele Millionen Gold verschaffen werde; die Hofkammer beantragte, obwohl sie durch solche Leute, die häufig Mißbrauch trieben, schon ganz scheu gemacht sei, de $m$ Manne doch ein Versicherungsdekret zu geben, doch müsse er zug leich sein arcanum eröffnen, und Leopold billigte diesen Rat (Hofkammerrefe rat exp. I5 Februar 1678 . Vermischte Vorschlage, Fasc Nr. I 8 976).

2) Vgl E. v. Ottenthal, Curialistische Finanzpläne fur K Leopold I. Mitteilungen des Instituts f. österr. Geschichtsforschung, I I. Band - Wegen des Urhebers, und da es für Sinzendorfs egoistischen Unternehmungsgeist bezeichnend ist, erwahne ich folgendes Projekt Als Becher 1665 in bayrischen Diensten nach Holland reiste, trat er mit einem gewissen Martin Elers in Verbindung, um eine Kompanie zur Grundung eines Kaufhauses in Munchen zu bilden, auf das Gutachten des Elers hin kam 'die Kompanie zustande, die sich alsbald auf die Seidenmanufaktur be- 
hier die ganze Reihe der Abstufungen vom Betrüger ${ }^{1}$ ) bis zum klardenkenden und ehrlichen Vertreter einer jungen Wissenschaft.

schränkte (vgl. v. Erdberg a. a. O. S. 40). In seinem „Polit. Discurs“ (Ausgabe von I688, S. 404) nennt Becher Elers noch einen klugen Mann in negotiis, in seiner ,närischen Weisheit" $\dot{S}$. 1 $43 \mathrm{f}$. erzählt er, Elers se "ein verunglückter Kaufmann", von Geburt ein Hamburger, , hat zwar nichts studiert, aber doch ein gutes Ingenium zu allerhand Vorschlagen und Concepten, womit er sich lange Jahre schleppt, aber wenig damit gefruchtet"; Elers habe das Seidenwattemachen in Europa erfunden, dann habe er dem Kurfursten von Mainz vorgeschlagen, er wolle allen rheinischen Wein aufkaufen, in Florenz wollte er zweimal im Jahre Seide erzeugen ,und andere Dinge mehr, die er zu Wien, München und Paris proponiert. Die Concepte sind wohl gut, und der Mann ist scharfsinnig genug, aber die Praxis ist ein anders als die Speculation", ein Wort, das ubrigens auf Becher selbst auch Anwendung finden kann. - I668 nun meldete sich dieser Elers, "der auch mit Kurmainz und Kurbayern in einem besonderen Kontrakt steht und als ein in Commerziensachen sehr beriihmter Practicus recommandirt ist" , in Wien mit Vorschlägen, durch die der Länder Einkommen nıcht allein ohne Beschwer der Untertanen, sondern mit großem Nutzen derselben vermehrt werden könne, und verlangte dagegen den Verlag, "erbliche Abfolgung " der Hälfte bzw. von zwei Dritteln des Gewinns der einzelnen Vorschlage, eine besondere Belohnung für jeden derselben, ferner Exemtion für seine Person und Familie von allen Landesanlagen und Forderungen, falls er sich in den kaiserlichen Erblanden niederlassen sollte, endlich Freiheit, äbnliche oder gleiche Kontrakte mit andern Fürsten und Herren $z u$ schließen. Er wollte, behauptet Sinzendorf, nicht mit der Kammer, sondern nur mit ihrem Präsidenten verhandeln und schließen. Sinzendorf erklärte, es falle ihm schwer, diesen Kontrakt wegen der zu erwartenden Spesen einzugehen, da er schon beim Seidenwesen große Auslagen gehabt habe, die ihm nicht ersetzt wurden; es wäre ihm lieber, wenn der Kaiser oder jemand anderes gegen Anteil am Gewinn das Geld vorstrecken wuirde da uberdies bei solchen privaten Kontrakten sich dann oft "allerhand ungleiche Nachreden zu ereignen pflegen". Nichtsdestoweniger bot er an, falls sonst niemand die Kosten decken wolle, "dem Lande zum Besten" die Sache auf sich zu nehmen, was Leopold billigte (Referat an den Kaiser 4. April I668, Haus-, Hof- und Staatsarchiv, Ad Hofkammer [aus der Kabinettskanzlei], Fasc. Nr. 142a). Nach einem Vermerke betrafen Elers' Vorschlage "Gewurzsachen," vermutlich einen Gewürzaufschlag, ein außerordentliches Mittel, das dann $168+$ wieder in Erwägung gezogen wurde (die anwesende an die hinterlassene Hofkammer 3. März I684, Hoffinanz, Fasc. Nr. 13 866). Über die Tätigkeit des Elers in Mưnchen vgl. M. Döberl, Innere Regierung Bayerns nach dem Dreißigjährigen Kriege, Forschungen zur Geschichte Bayerns, I2. Bd., S. 66 ff.; tiber seine Wirksamkeit am Mainzer Hofe G. Mentz, Johann Philipp v. Schönborn, Kurfurst von Mainz, 2. Bd. (Jena r899), S. I 40 f.

1) In einem charakteristischen Falle bewahrte der ausgezeichnete Hofkammerpräsident Christoph Freiherr v. Abele das Ärar vor schwerer 